\title{
Establecimiento y Propagación del Darwinismo en Chile: Recepción y Elaboración de las Ideas
}

\section{The Settlement and Spread of Darwinism in Chile: Reception And Elaboration of Ideas}

\author{
Rodrigo Medel \& Alberto Veloso ${ }^{1}$ \\ Departamento de Ciencias Ecológicas, Facultad de Ciencias, \\ Universidad de Chile, Casilla 653, Santiago, Chile. \\ Email: rmedel@uchile.cl
}

\begin{abstract}
RESUMEN
En este trabajo presentamos un análisis del escenario social y cultural de la época en que ocurre el viaje de Darwin a Chile. Sobre esa base, describimos el impacto a corto y mediano plazo del viaje del naturalista en el ambiente cultural de la época en el país, sugiriendo algunos elementos que pudieron haber influido en el establecimiento y propagación de las ideas Darwinianas en el Chile de fines del siglo XIX y primera mitad del siglo XX. Identificamos tres etapas en el proceso. Primero, una etapa naturalista, relacionada con el aporte directo de Darwin al conocimiento de la diversidad biológica de Chile descrita hasta aquel momento. Segundo, una etapa ideológica, caracterizada por debates no científicos de su obra, los cuales fueron efectuados por intelectuales chilenos interesados en los alcances religiosos, éticos, sociales y educacionales de la obra del naturalista. Tercero, una etapa científica, relacionada con la implementación de investigación formal en biología evolutiva impulsada por el desarrollo de la Síntesis Moderna a comienzos del siglo XX. Desde entonces, la investigación sobre biología evolutiva Darwiniana ha aumentado en amplitud y profundidad y continúa expandiéndose a través de nuevos y diversos programas de investigación.
\end{abstract}

Palabras Clave: Beagle, Darwin, historia natural, selección natural.

\begin{abstract}
We analyze the social and cultural scenario prevalent at the moment of Darwin's visit to Chile. On this basis, we describe the short-and mid-term impact of the Beagle voyage upon the cultural environment at that time, and suggest some elements that may have influenced the settlement and spread of Darwin's ideas at the end of the 19th and first half of the 20th centuries in Chile. We identified three stages in the process. First, a naturalist stage, related with the direct impact of Darwin's work on the knowledge of biological diversity described in Chile at that moment. Second, an ideological stage, characterized by extra scientific debates on Darwin's major ideas. Most of this debate was carried out by Chilean intellectuals, which focused upon the religious, ethical, social, and educational consequences of Darwin's ideas rather than in their scientific soundness. Third, a scientific stage, related with the implementation of formal research in evolutionary biology impulsed by the development of the Modern Synthesis in the first half of the $20^{\text {th }}$ century. Since then, research on evolutionary biology has increased in scope and depth and continues spreading through diverse and new research programs in the country.
\end{abstract}

KeYwords: Beagle, Darwin, natural history, natural selection.

${ }^{1}$ Orden de los autores decidido al azar.

Pp. 7-18 en D’Elía, G. 2009. Una celebración de los aniversarios darwinianos de 2009. Gayana 73 (suplemento): 1-88. 


\section{INTRODUCCIÓN}

La génesis y nacimiento de la Teoría de Evolución por Selección Natural (TESN desde aquí en adelante) es uno de los eventos más importantes en la historia de la ciencia, comparable solamente a las revoluciones asociadas a los nombres de Copérnico, Lavoisier, Newton y Einstein. La revolución Darwiniana no sólo consistió en la emergencia de una teoría integrativa de fenómenos hasta entonces no articulados entre sí, sino que representó una profunda transformación de la manera de concebir el mundo natural. Ningún cambio de esa magnitud, sin embargo, emerge de manera instantánea sin antecedentes previos. Existe siempre, de acuerdo a Kuhn (1962), una etapa previa caracterizada por la ausencia de un cuerpo doctrinario sólido capaz de orientar las investigaciones y dar sentido a las observaciones. Es así como con anterioridad a Darwin, el conocimiento de historia natural, taxonomía, embriología, anatomía comparada, geología, paleontología, botánica y zoología, entre otras, se encontraban en una etapa descriptiva y clasificatoria. Darwin fue probablemente uno de los últimos científicos que abarcó campos tan extensos del conocimiento biológico y geológico, los que posteriormente se subdividieron en disciplinas y subdisciplinas a medida que se acumulaban los conocimientos (Eldredge 2005). El mérito de la proposición de Darwin fue articular por primera vez las diversas piezas de información provenientes de las disciplinas antes mencionadas en un marco conceptual unificado, coherente y con un alto contenido empírico. Esto no significa que durante el período pre-Darwiniano, las disciplinas estuvieran desprovistas de conceptos. Por el contrario, muchos de los términos acuñados por los científicos de aquella época continúan siendo válidos hasta el día de hoy (e.g., las clasificaciones binominales Linneanas). Sin embargo, tales conceptos no fueron capaces por sí solos de dar origen a un paradigma Kuhniano ya que por lo general se articulaban en torno a ideas extracientíficas, tales como elementos de la religión predominante. Es así como si bien existieron intentos por proponer teorías coherentes de cambio natural antes de Darwin, por lo general tales proposiciones requirieron visiones alusivas a la creación para ser socialmente justificables.

Los cambios de paradigma cambian la concepción del mundo. Sin embargo, la asimilación de los nuevos paradigmas es contingente a las circunstancias sociales de cada región. La publicación de "On the Origin of Species by Means of Natural Selection, or the Preservation of Favoured Races in the Struggle for Life" en 1859 (El Origen de las Especies desde aquí en adelante), enciende una intensa controversia en Europa sobre el papel de la selección natural como mecanismo del cambio evolutivo, desafiando la tradición social, la moralidad y religión (e.g., Hodge 1874). Darwin demuestra que no es necesario recurrir a lo sobrenatural para explicar la diversidad de la vida. Si bien el naturalista no se pronuncia en forma definitiva sobre el origen del hombre, esta discusión la pospone en atención a que el ambiente científico existente y los prejuicios de la sociedad de la época pudieran llegar a entorpecer el desarrollo de la TESN. La publicación de El Origen de las Especies tuvo un enorme impacto en el pensamiento científico de la segunda mitad del siglo XIX, siendo adoptada por un grupo numeroso de científicos de la época, entre ellos Thomas Huxley (1825-1895) en Inglaterra y Ernst Haeckel (1834-1919) en Alemania, entre muchos otros. La aceptación de Darwin y la TESN fuera de Europa dependió de las contingencias históricas y sociales de cada region y es un proceso que se encuentra aún en curso.

En este trabajo, analizaremos globalmente el impacto de Darwin en el escenario cultural chileno. Para ello, examinaremos el contexto general en que aparece la obra del naturalista para posteriormente examinar con más detalle el escenario político y social prevaleciente en Chile al momento del viaje del HMS Beagle, y finalmente evaluaremos el impacto a corto y mediano plazo que la visita de Darwin y su TESN han tenido sobre generaciones de intelectuales en el país. No es objetivo de este trabajo el efectuar una descripción de la extensa obra científica de Darwin, ni tampoco relatar cronológicamente la experiencia del naturalista en Chile (para un análisis de tales aspectos véanse las contribuciones de Papp 1977, Márquez Breton 1982, Yudilevich \& Castro 1996, Bacigalupo \& Yudilevich 1998 y Urzúa 2009), sino más bien bosquejar algunos elementos que a nuestro juicio han moldeado el establecimiento y desarrollo de algunas de las ideas Darwinianas en nuestro país. 


\section{LA OBRA DEL NATURALISTA}

La vocación científica de Charles Darwin (18091882), tomó una orientación definitiva a los 22 años cuando el naturalista efectúa un viaje alrededor del mundo en el buque británico HMS Beagle entre los años 1831-1836. El trabajo de Darwin a bordo consistió principalmente en la recolección de ejemplares de la fauna y flora actual y extinta, conjuntamente con observaciones geológicas de los distintos puntos de recalada en los continentes e islas tocados en su itinerario. En su calidad de naturalista, el destino de los especímenes recolectados eran los museos y exhibiciones que proliferaban en gran parte de Europa y especialmente en Londres. Las evidencias obtenidas por Darwin en el viaje del Beagle y sus reflexiones sobre la naturaleza de sus hallazgos fueron consignadas en su diario de viaje y condensadas en su libro Journal of Researches, conocido como The Voyage of the Beagle en 1839 (1844 por Murray). Tales piezas de información fueron fundamentales para la elaboración posterior de su TESN. Destacan entre las observaciones, aspectos de morfología (incluida la osteología) y distribución geográfica de especies fósiles y actuales en las costas de Sudamérica. Estas observaciones permitieron a Darwin argumentar acerca del origen de las especies y su cambio natural en base a relaciones de parentesco en contraposición a la interpretación creacionista, visión prevalente en aquella época. Llamó la atención del naturalista el que especies de ñandúes similares se sucedieran latitudinalmente y en estrecha proximidad geográfica (Rhea americana y Pterocnemia pennata). Asimismo, las similitudes morfológicas de diseño entre especies extintas y actuales le sugirieron la existencia de vínculos ocultos de parentesco. Por ejemplo, los fósiles encontrados en expediciones por la Patagonia de perezosos terrestres del tamaño de un elefante (Megatherium) y otros fósiles con estrechas relaciones morfológicas con formas actuales tales como el de un armadillo gigante (Glyptodon) semejante a los actuales armadillos Euphractus, endémicos de Sudamérica, fueron observaciones cruciales en la corroboración de las ideas de transformismo (Eldredge 2009). Durante el transcurso de su viaje en el Beagle, Darwin tiene acceso a la sexta edición de un ensayo sobre población humana del economista Thomas Malthus publicado inicialmente en 1798, quien plantea el inevitable colapso de la sociedad industrial por la relación existente entre la escasez de recursos y el crecimiento poblacional. Darwin aplica estas ideas al campo de las ciencias naturales y da con la TESN, donde expresa que a medida que aumenta el número de individuos en las poblaciones, los recursos disponibles disminuyen y se produce una lucha por la existencia. Es así como los organismos con rasgos favorables para enfrentar el medio en que habitan eran quienes sobrevivían y dejaban una mayor descendencia en comparación a otros individuos, transmitiendo así sus rasgos ventajosos a las generaciones futuras.

A su vuelta a Inglaterra, en 1836, Charles Darwin inicia la catalogación final y supervisión de los estudios taxonómicos de los especímenes recolectados en el HMS Beagle que habían sido previamente encargados a notables naturalistas y paleontólogos de la época. Entre éstos, es necesario destacar los trabajos de Leonard Jenyns en peces, Thomas Bell en anfibios y reptiles, John Gould en aves, George Waterhouse en mamíferos, Thomas Hooker en plantas y Richard Owen en mamíferos fósiles. Como resultado de sus observaciones de gabinete y correspondencia sostenida con naturalistas de todo el mundo, la que se extiende por más de 20 años, Darwin reúne y organiza la evidencia para su idea que las especies no eran fijas sino que se transmutaban unas en otras a lo largo del tiempo. En El Origen de las Especies, considerada su obra maestra, desarrolla la TESN, la que provee un mecanismo para la transformación de las especies. Se trata de una simple y elegante explicación para la complejidad y diversidad de la vida en la Tierra, que modificó para siempre la visión del mundo natural y la concepción del lugar que en él ocupamos. A diferencia de la breve temporalidad ocupada en las prácticas de los criadores y de la selección artificial, el cambio evolutivo en la naturaleza se desarrolla en forma natural y gradual, requiriéndose de un horizonte de tiempo mayor para observar la transformación de las especies.

\section{EL ESCENARIO SOCIAL DE LA ÉPOCA}

Para comprender el escenario histórico y social en que se lleva a cabo el segundo viaje del HMS Beagle es necesario retrotraerse a la situación económica y social de la primera mitad del siglo XIX en Europa. A comienzos del siglo XIX se inicia la revolución industrial europea en que no sólo se producirían 
manufacturas sino también bienes. Producto de la creciente producción industrial, fue necesario incorporar nuevos mercados compradores de los artículos elaborados y al mismo tiempo importar materias primas y alimentos. América Latina presentaba buenas condiciones para ello, salvo por la existencia de un monopolio comercial impuesto por España, país que controlaba las importaciones y exportaciones desde y hacia las colonias del nuevo mundo. El Chile colonial de entonces, al igual que el resto de los países de Latinoamérica, desarrolló una economía de exportación cuya función primordial era proveer de materia prima al mercado mundial. Cincuenta años antes, Inglaterra ya había iniciado un ambicioso plan de expansión que la condujo a conquistar algunos territorios latinoamericanos y a alentar la independencia de las colonias de España y así copar los nuevos mercados con su producción industrial en ascenso. Una vez ocurrida la independencia de Chile y rotos los lazos con España, se ampliaron los contactos del país con otros países de Europa y con los Estados Unidos. Las metrópolis europeas, que se encontraban en pleno proceso de revolución industrial, aseguraron buenos precios a las materias primas. A cambio, Chile debía asegurar el ingreso de manufactura extranjera a bajas tasas arancelarias. Es así como se otorgaron las garantías exigidas por las potencias extranjeras y Chile fue incorporado al mercado mundial en formación, desarrollándose acorde a un papel de país abastecedor de materia prima e importador de artículos elaborados por la industria europea (Vitale 1991).

En los años que siguieron a la independencia de Chile, ocurrió un período de amplia inestabilidad política. La fuerte presión que ejerció la aristocracia criolla por recuperar los beneficios perdidos luego de la independencia de España llevó a O'Higgins a presentar su renuncia al cargo de Director Supremo en 1823 (Figura 1). El vacío de poder generado por la renuncia de O'Higgins originó un período caracterizado por una serie de intentos de organización republicana. Fue así como se elaboraron varias constituciones destinadas a organizar los poderes del Estado emergente. Las características más relevantes de esta etapa son los intentos de organizar políticamente a la nueva república bajo términos políticos e ideológicos influenciados por la Ilustración y la Revolución Francesa. Mientras tanto, la dependencia de la economía con
Inglaterra se hacía más fuerte, mediada a través de préstamos blandos a condición de abrir las compuertas comerciales para instalar productos ingleses manufacturados. El gobierno criollo contrajo una serie de compromisos económicos que generalmente no podía cumplir, lo cual era tolerable a cambio de crecientes franquicias comerciales con el prestamista. Inglaterra fue la nación que otorgó mayor número de préstamos, lo cual promovió la llegada de ingleses al país a efectuar labores de administración de las fuentes de riqueza y a promover el comercio local de los productos manufacturados en Europa. Este hecho no sólo fue privativo a Chile sino que incluyó a la mayor parte de los países en proceso de independencia en Sudamérica, lo cual constituyó, a juicio de historiadores, la razón última de la inspección y reconocimiento de los mares de Sudamérica (Urzúa 2009).

El estado chileno se encontraba sin divisas producto de la alta inversión en armamento para la defensa de amenazas realistas, las que se sabía habían ya cobrado expresión en frustradas arremetidas por parte de grupos internos en el Río de la Plata y Perú. No obstante, en el período se plantearon importantes iniciativas sociales, tales como la abolición definitiva de la esclavitud en 1828, medidas que fueron resistidas por la aristocracia local, acelerando las condiciones para el estallido de la guerra civil de 1829-1830 (Figura 1). La guerra civil permitió unificar la ideología conservadora en un sólido frente que aplastó las ideas democráticas y liberales. La ideología conservadora, ahora dominante, se expresó en la necesidad de un gobierno autoritario, centralizado y elitista que asegurara los intereses de los latifundistas y comerciantes, y especialmente la exportación de las materias primas hacia Inglaterra. Es así como se consolidaron los intereses económicos de una burguesía criolla en expansión en estrecha asociación con capitales ingleses. Luego de la guerra civil, en 1831 fue electo Joaquín Prieto (1831-1836 y 1836-1841) para la primera magistratura y Diego Portales para Vicepresidente. Fue la institucionalización del autoritarismo. Uno de los objetivos del nuevo orden fue desarticular intentos liberales, respaldar proyectos económicos con potencias extranjeras, y proponer un texto constitucional que vio la luz en 1833, en el cual se dotaba al Presidente de facultades extraordinarias para imponer la normalidad en casos de desórdenes 


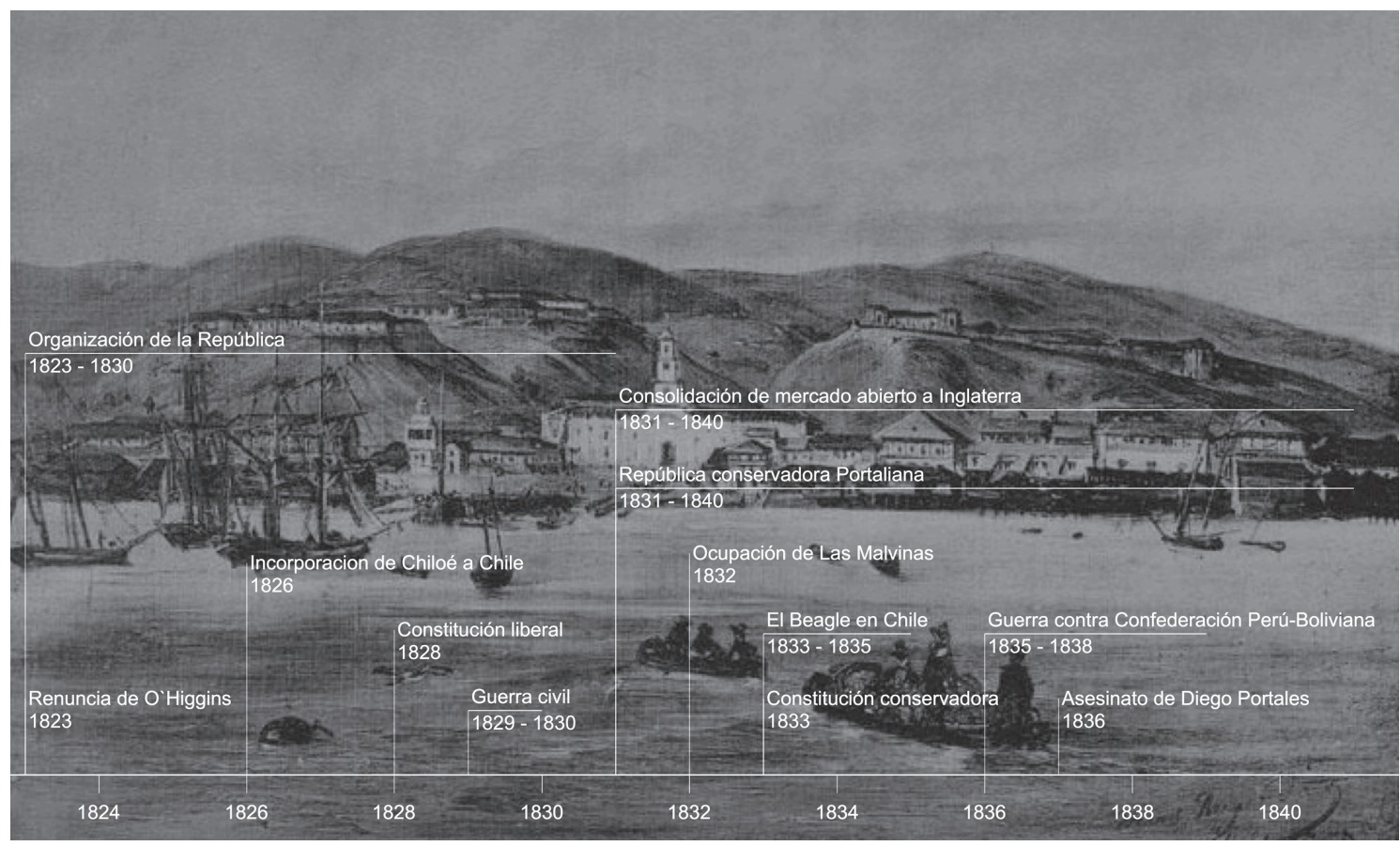

FIgURA 1. Esquema cronológico de los eventos históricos y sociales más importantes acontecidos en la época del viaje de Darwin por Chile.

FIGURE 1. Chronological outline of the most important historical and social events that occurred at the time of Darwin's travel in Chile.

y agitaciones, acallándose la prensa política durante los 10 años venideros. Es en este escenario político autoritario, de alta dependencia económica con Inglaterra y de expansión imperialista inglesa, en que ocurre la segunda visita del Beagle a Chile.

Los límites geográficos de la República al momento del viaje de Darwin eran poco precisos, aunque incluían prácticamente la totalidad de la que actualmente es la Patagonia Argentina. Es así como la Constitución de 1833, impulsada por el gobierno de Prieto, dictó la necesidad de precisar los límites geográficos del país. Los territorios septentrionales eran prácticamente desconocidos y tenían significado teórico pero no práctico. Esta instrucción, permitiría además de ganar conocimiento del territorio, controlar la expansión imperialista de Inglaterra por lugares estratégicos en América del Sur. Las Islas Malvinas ya habían sido ocupadas por Inglaterra en 1832 (Figura 1). Tierra del Fuego aún no formaba parte de Chile. Solamente en 1843, diez años después del viaje de Darwin, el gobierno de Manuel Bulnes incorporó formalmente el Estrecho de Magallanes y las islas circundantes al territorio nacional.

En la década de 1830 la minería de oro ya había decaído, pero al mismo tiempo la minería de plata experimentaba un gran impulso debido al descubrimiento del mineral en Arqueros, lo cual incentivó la exploración detallada de la red montañosa entre La Serena y Copiapó (Villalobos et al. 1974), avalada por una ley española que entregaba licencia inmediata de explotación a quienes descubrieran una mina previo pago de cinco chelines (Urzúa 2009). En el Viaje del Beagle, Darwin, al visitar el centro de Chile en 1834 (Figura 2), indica: "Casi todos los cerros han sido perforados con el fin de descubrir minas de oro: el furor de la minería no ha dejado prácticamente lugar en Chile sin explorar" (Agosto 17, 1834, p. 254). La dependencia económica respecto a Inglaterra era muy manifiesta y las situaciones de empobrecimiento de los mineros no escaparon a las observaciones de Darwin, quien indicó: "Los mineros trabajan muy duro. Se les otorga poco tiempo para comer, comienzan a trabajar apenas hay luz y terminan al oscurecer. Se les paga 
una libra esterlina al mes... Ellos rara vez comen carne ya que con 12 libras por año, se deben vestir y sostener a sus familias" (Agosto 18, 1834, p. 256). No obstante que la economía chilena se basaba en la exportación de materias primas, al tiempo de la visita de Darwin existían focos rurales de economía agricultural que se sustentaban por sistemas semi feudales. Darwin observa este fenómeno en los alrededores de San Fernando (Figura 2), donde indica: "La condición de los agricultores es peor que la de los mineros. Sus salarios son más bajos y viven casi exclusivamente de porotos. Esta pobreza se debe atribuir al sistema de tipo feudal en que la tierra es trabajada: el propietario otorga una pequeña porción de tierra al campesino y a cambio posee sus servicios por el resto de la vida, sin salario... La extrema pobreza es muy común en la clase trabajadora de este país." (Septiembre 13, 1834 , p. 263). Este conspicuo rasgo social, cuyos orígenes se pueden trazar hacia la época colonial en que las instituciones creadas facilitaron una estructura en que unos pocos individuos recibieron facilidades para explotar recursos mineros y agrícolas con abundante mano de obra, deprivando a las poblaciones originarias del acceso a las fuentes de riqueza, se había perpetuado hacia un sistema de hacienda avalado por la Constitución de 1833, consolidando de este modo un sistema de diferenciación social (Salazar \& Pinto 1999). Si bien el escenario social y político al momento de la segunda visita del Beagle contenía fuertes rasgos coloniales, con adhesión a un orden jerárquico, autoritario y católico, una vez terminado el segundo gobierno de Prieto, la educación contuvo elementos más racionalistas que condujeron a adoptar posturas más secularizantes o anticlericales en oposición a la doctrina católica. De esta manera, el debate ideológico durante la segunda mitad del siglo XIX giró en torno a la dicotomía catolicismo versus laicismo, lo cual influyó de manera determinante en la aceptación y establecimiento de las ideas de Darwin en Chile. El año del regreso del Beagle

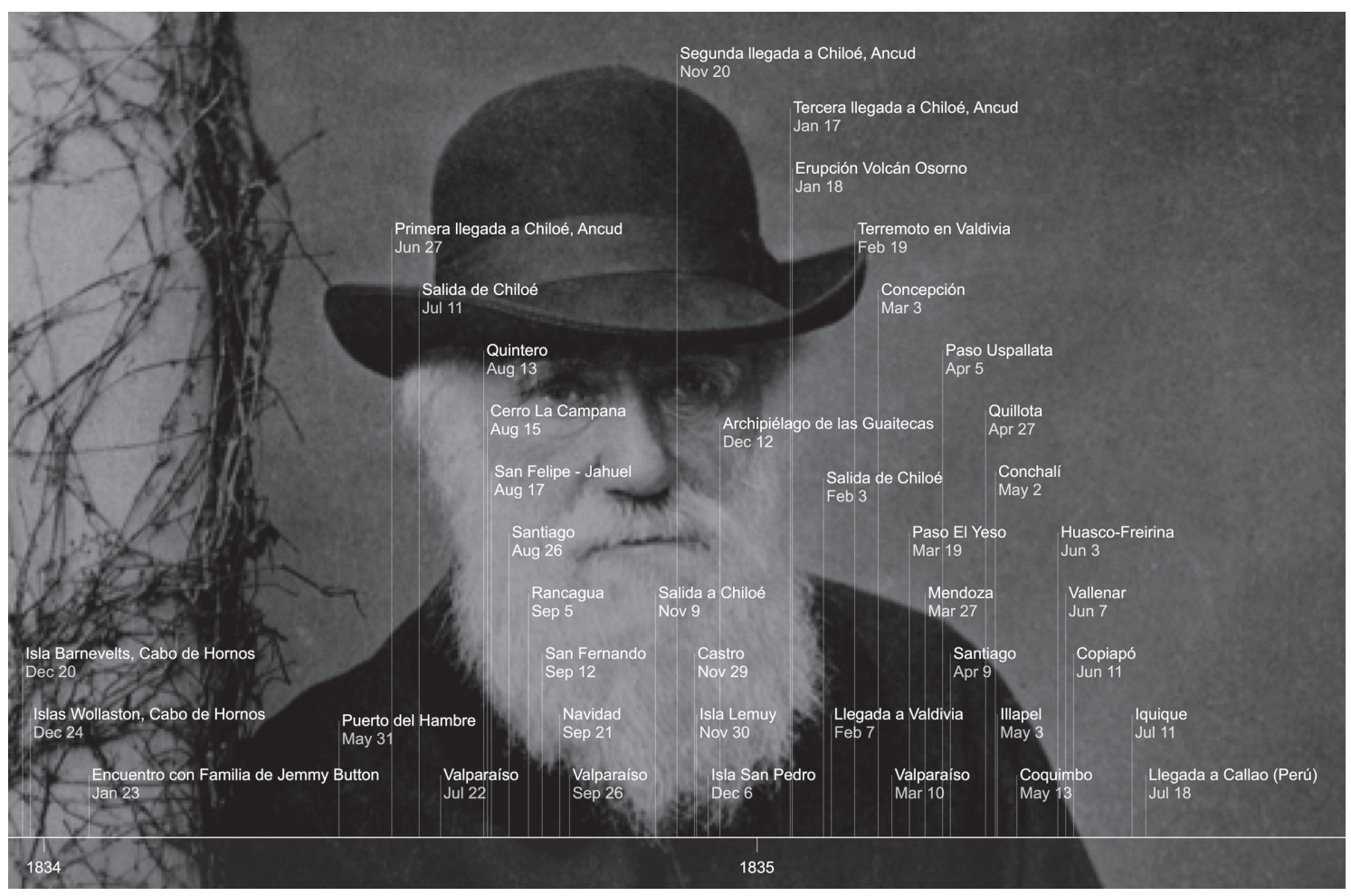

FIgURA 2. Esquema cronológico de las visitas efectuadas por Darwin a distintas ciudades de Chile.

FIgURE 2. Chronological outline of the visits made by Darwin to different cities in Chile. 
a Inglaterra, en 1836, estalla la guerra de Chile contra la Confederación Perú-Boliviana. Si bien las razones últimas del conflicto descansan en políticas expansivas por parte de la Confederación y de una política de aseguramiento del orden interno mediante la ideología de la guerra externa, acción propiciada por el orden Portaliano, los resultados armados del conflicto no alcanzaron al viaje del Beagle (Figura 1).

\section{EL IMPACTO DE DARWIN EN CHILE}

\section{La Etapa Naturalista}

Es posible distinguir tres etapas en el proceso de asimilación de las ideas Darwinianas en Chile. En primer lugar, ubicamos una etapa que denominaremos etapa naturalista que está referida a las contribuciones naturalistas inmediatas de Darwin en su paso por el país. Los efectos de las investigaciones de Darwin en Chile se suman al cúmulo de evidencias y comprobaciones in situ de los argumentos sostenidos por el propio naturalista más adelante en su teoría evolutiva. Estos tienen que ver en primer lugar con contribuciones relacionadas al incremento de información disponible sobre flora, fauna y geología del país, incorporando detallada información sobre regiones del territorio escasamente conocidas hasta aquel momento. Ya desde 1820 los gobiernos de Chile contrataban a naturalistas extranjeros para explorar y conocer el territorio y consolidar la república. En este sentido, el aporte de Darwin al conocimiento de la diversidad biológica de Chile complementa la labor naturalista de científicos que lo precedieron, entre otros del abate Juan Ignacio Molina (1740-1829) y Claude Gay (1800-1873) (Figura 3). El trabajo de Gay ya llevaba 4 años de desarrollo al momento de la llegada de Darwin a Chile. En este sentido, Darwin recoge el conocimiento de Gay y se embebe de diversos aspectos de la historia natural chilena mediante conversaciones directas con el naturalista

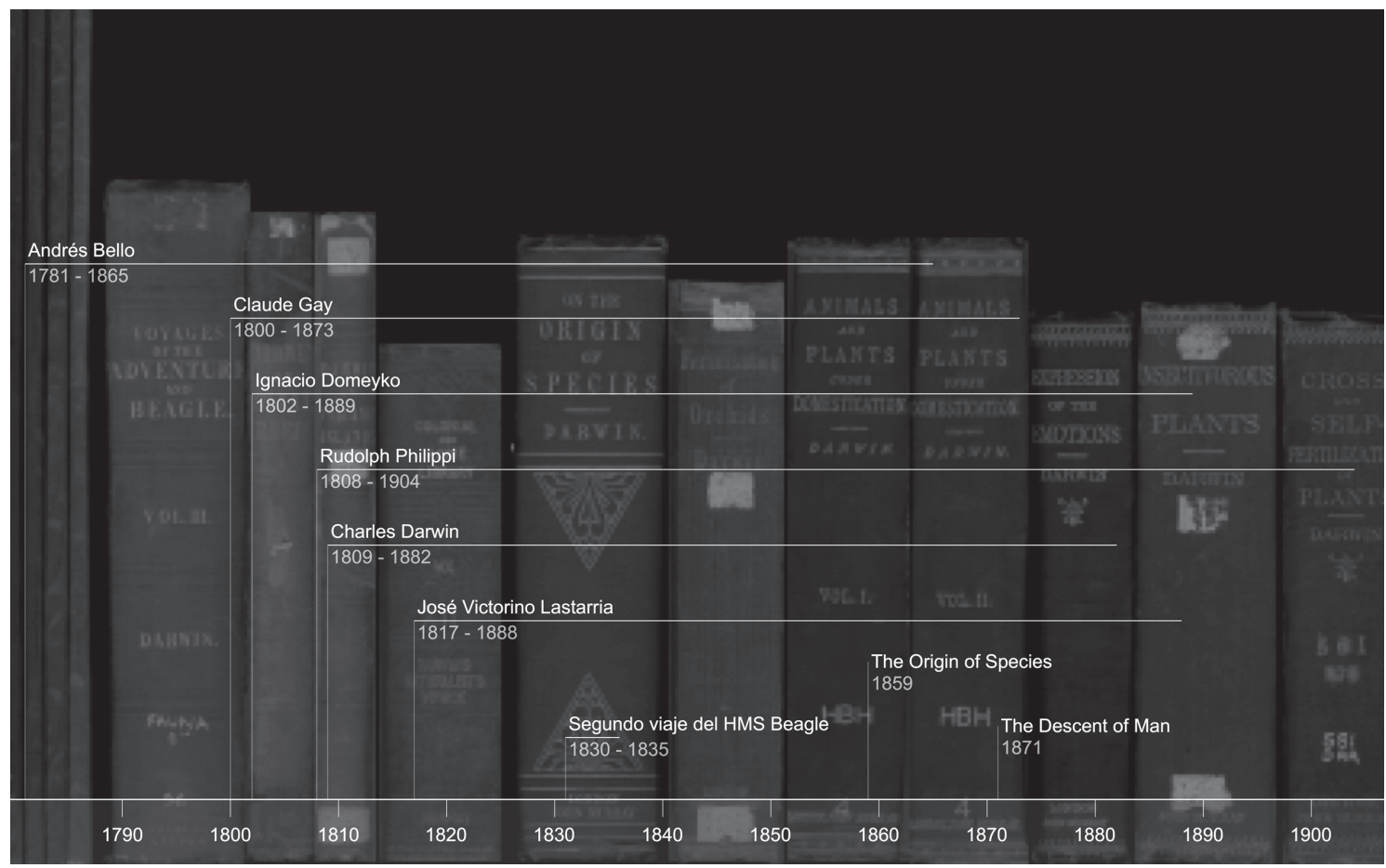

FIGURA 3. Esquema cronológico de los principales autores asociados a la discusión de las ideas Darwinianas en Chile en la segunda mitad del siglo XIX.

FIGURE 3. Chronological outline of the main authors involved in the discussion of Darwin's ideas in the second half of the $19^{\text {th }}$ century in Chile. 
francés como tambien a través de la lectura de sus escritos publicados en Annales des Sciences (Urzúa 2009). La nueva información recopilada por Darwin respecto a la fauna, flora y geología de Chile es celebrada por los naturalistas y fuertemente apreciada por los gobiernos de la época, interesados en conocer y afianzar la soberanía de Chile en todos los territorios de la naciente república. Es así como la misión de Darwin fue reconocida por las autoridades de gobierno de la época. Se le proporcionó un salvoconducto otorgado por el Presidente José Joaquín Prieto, “...ordeno a los intendentes de la provincias, los gobernadores, jueces, por cuyos territorios transitare y operare no pongan al expresado naturalista el menor embarazo, antes bien le protejan y ayuden en cuanto penda de su arbitrio para el mejor éxito de sus interesantes operaciones" (Yudilevich \& Castro 1996).

Las descripciones realizadas por Darwin en territorio Chileno fueron compiladas por Andrés Bello y publicadas en el periódico El Araucano a tan sólo 3 años luego que el Beagle retornara a Inglaterra en 1836. Se trata de la primera referencia directa al viaje del naturalista realizada por un intelectual local. En publicaciones sucesivas, la información recopilada por Bello se refiere a geología, terremotos, flora, fauna y especímenes fósiles, descripciones de territorios prácticamente desconocidos como la Patagonia, los bosques de Valdivia y Concepción, el desierto de Atacama, así como descripciones de usos y costumbres de la población humana de diferentes regiones de Chile (Bacigalupo \& Yudilevich 1998). La selección de estos temas obedece a lo novedoso que ellos resultan para el conocimiento de la diversidad biológica y cultural de Chile y al mismo tiempo por un sentido utilitario de los mismos, ya que son empleados como complemento de esfuerzos educativos y de gestión por parte de las autoridades nacionales progresistas de la época.

En la narrativa del Viaje del Beagle, Darwin (1845) deja consignadas observaciones sobre aspectos relacionados con la diversidad biológica de Chile y la distribución geográfica de elementos de la flora y la fauna. Es así como los nuevos hallazgos se agregan al gran número de evidencias obtenidas en el periplo de la embarcación por distintos continentes e islas, los que forman parte importante de las evidencias utilizadas como fundamento del proceso evolutivo y de su TESN. Con sus observaciones,
Darwin realza la variabilidad de las especies y enfatiza la influencia del clima como explicación de esta variabilidad. Se trata de respuestas adaptativas de los seres vivos, incluyendo al hombre, a extremos climáticos, con escasez de recursos alimentarios y por ende en "lucha por la existencia". Sin embargo, probablemente fueron los eventos de vulcanismo y movimientos tectónicos experimentados por Darwin, así como la presencia repetida de fósiles encontrados en diversas regiones de Chile, los que influyeron de manera decisiva en su concepción transformista de las especies en contra de la concepción tipológica y fijista de las mismas. El hallazgo repetido de fósiles en bordes costeros es utilizado por Darwin para demostrar los fenómenos de extinciones locales y para desarrollar su teoría de formación de montañas, un aspecto de su obra inspirada por el primer volumen de los Principos de Geología de Lyell, libro que llevaba consigo en el viaje del Beagle. En la costa de Coquimbo, Darwin encontró la mejor evidencia geológica para articular sus ideas geológicas (Eldredge 2009). Las capas de sedimentos con fósiles invertebrados marinos le revelaron una secuencia cronológica hasta el presente a medida que sus observaciones iban desde los estratos inferiores a los superiores. Sin embargo, Darwin no se refiere a esta observación en el contexto del transformismo, sino que se remite a discutir el aumento proporcional de fósiles de especies contemporáneas hacia los estratos superiores del terreno. Las comparaciones morfológicas entre las especies (actuales o fósiles) y su distribución geográfica fueron interpretadas con precaución, aunque constituyeron parte importante en su posterior elaboración de la existencia de relaciones de parentesco entre taxa. Si bien es cierto que Darwin no continuó profundizando sus ideas geológicas inspiradas en Chile, y suponiendo que no hubiera propuesto la TESN, su aporte al conocimiento geológico lo habrían hecho ocupar un lugar destacado tanto por sus observaciones en el campo como por sus ideas sobre la edad de la Tierra (Eldredge 2005).

\section{La Etapa Ideológica}

En segundo lugar, se desarrolla una etapa relacionada con la apreciación y debate de las ideas de Darwin en círculos intelectuales del país, que denominaremos etapa ideológica. Las investigaciones realizadas por Darwin fueron objeto de numerosas publicaciones y tanto los resultados obtenidos y relatados en el 
"Viaje del Beagle" así como en su obra fundamental "El Origen de las Especies" y su posterior "Origen del Hombre" y otras contribuciones, constituyen una obra unitaria, que tan sólo con el objeto de entender los hechos y conmociones sociales, tanto científicas como políticas que ellas suscitaron al momento de ser publicadas, pueden ser objeto de desglose en términos cronológicos. Tal como se mencionó previamente, la formalización final de la TESN se lleva a cabo con la publicación de El Origen de las Especies en 1859. En esta publicación, considerada su obra capital, Darwin incorpora las ideas de Malthus (1826), y da con la selección natural como un mecanismo explicativo del proceso evolutivo. Lo revolucionario de las ideas de Darwin hace que éstas tengan repercusiones en diversos países del Hemisferio Norte, principalmente europeos, y también en Chile. En ambos escenarios, tanto el internacional como el nacional, se discuten sus ideas con vehemencia, tan sólo separadas por el desfase propio de las comunicaciones de la época. Sin embargo, mientras el debate suscitado en Europa y los Estados Unidos de Norteamérica se basa en el análisis por pares en lo relacionado al mérito científico de las ideas de Darwin, en Chile esta discusión está ausente o es prácticamente inexistente, reservándose a una élite reducida de intelectuales de la época donde confluyen concepciones filosóficas opuestas y aspectos no contenidos o poco desarrollados por la ciencia hasta esos momentos (Márquez Breton 1982). Gran parte de tales discusiones fueron efectuadas por los hijos de los participantes de la revolución liberal de 1820 (Figura 3). Esta nueva generación se constituía por intelectuales ilustrados y particularmente receptivos a las corrientes culturales de Europa, en especial al positivismo Comtiano. Fueron ellos los encargados de apreciar y difundir los movimientos de opinión que se propagaban por todo el mundo tales como el liberalismo doctrinario y posiciones anticlericales. Curiosamente, la interpretación Darwiniana mecanicista del transformismo de las especies no fue fácilmente aceptada por los naturalistas locales, si bien se señalaba que la TESN contenía un gran número de evidencias que era necesario analizar en su mérito científico. De hecho, es una ironía de la historia que el naturalista Philippi, quien a pesar de no creer en el transformismo, por el hecho de haber sido el primero en mencionar marginalmente las ideas de Darwin en un texto de estudio en 1866, hubiera sido duramente atacado y denostado por la prensa religiosa y conservadora de la época. Fue la primera manifestación pública en contra de las ideas de Darwin en el país. Nosotros coincidimos con Márquez Breton (1982), quien señala: "En el caso específico de Chile, la introducción de las ideas Darwinianas se produce a través de un debate no de corte científico estricto. Se centra en las posibles conclusiones teóricas-religiosas, éticas o sociales. No hay discusión profunda sobre el tema biológico. Los temas que se discuten son asuntos religiosos, tolerancia, libre examen y otros semejantes". Es así como el debate estuvo a cargo principalmente de historiadores, sociólogos y educadores tales como Andrés Bello, José Victorino Lastarria, Diego Barros Arana y Valentín Letelier entre otros, siendo los científicos más bien conservadores y escépticos a las ideas de Darwin (Márquez Breton 1982).

Respecto a las contribuciones geológicas de Darwin, éstas estuvieron fuertemente influenciadas por su correspondencia con el geólogo Lyell, quien sostenía que las fuerzas que actualmente moldean la corteza terrestre serían las mismas que estuvieron presentes en el pasado y que gradualmente conformaron los paisajes actuales. Esta idea de gradualismo en los cambios de la corteza terrestre, que requería un mayor horizonte de tiempo que el invocado por los procesos de creación de la génesis bíblica, es adoptada por Darwin. Las observaciones geológicas realizadas por Darwin, incorporadas en el relato del Beagle, y en "Geological Observations on South America" (1842-1846) fueron poco conocidas o escasamente referidas por parte de los geólogos chilenos. Sólo existe una pequeña referencia por parte de Ignacio Domeyko (1874) y un desarrollo mayor de ideas en relación al solevantamiento de la costa de Chile (Del Barrio 1855). Esta apatía intelectual llama fuertemente la atención de Fuenzalida (1934), quien en su estudio historiográfico señala ignorancia o poco interés por las ideas del naturalista por parte de los estudiantes chilenos, no obstante que las ideas de Darwin ya habían sido aceptadas por la mayoría de los geólogos contemporáneos.

Un aspecto relevante de esta etapa lo constituye la proyección de su Teoría de la Evolución a la enseñanza de las Ciencias Naturales. Destaca en ello la obra realizada por Philippi (Figura 3), quien con su formación europea estaba al tanto de la importancia de la historia natural y de la necesidad de desarrollar una estructura museológica 
donde albergar colecciones sistemáticas. Esta labor tendría efectos culturales en educación primaria, escuelas y liceos, así como también en las universidades encargadas de la formación de profesionales. No obstante, en la enseñanza de la historia natural en escuelas y universidades, las reflexiones de Darwin sobre mutabilidad de las especies y principalmente su TESN, tropiezan con una reacción de sectores de orientación religiosa y conservadora, que se ubican en una trinchera opuesta, viendo a las nuevas ideas como un intento de sustitución de verdades reveladas por un ser superior. Los temores esgrimidos por este sector son que estas nuevas ideas puedan llevar a una degradación de la humanidad y se conviertan en un germen subversivo en el orden social existente. Las opiniones se dividen entre intelectuales de la francmasonería, por una parte, partidarios de incorporar las ideas evolucionistas y por otra por intelectuales y clérigos católicos que representan a sectores conservadores de la sociedad chilena. En los organismos de gobierno del país las posiciones adoptadas tienen consecuencias en los planes de estudio de la instrucción pública y privada, llegando a situaciones en las que programas previamente aceptados en el sistema educacional sufren mutilaciones por decreto, proscribiéndose la enseñanza de la evolución en los programas oficiales de la enseñanza media (Tamayo 2004; Tamayo \& González 2005).

\section{La Etapa Científica}

En tercer lugar, se desarrolla una etapa relacionada con el establecimiento y diseminación de la investigación científica en biología evolutiva que denominaremos etapa cientifica. Con posterioridad a los trabajos de Darwin, el avance científico global proporciona las piezas de información ausentes en su TESN, las cuales hacían referencia a los mecanismos responsables del tempo y modo de la evolución y especiación. Recién en el siglo XX los científicos combinan la evolución con la genética gracias, en parte, al redescubrimiento de los trabajos experimentales de Gregor Mendel sobre variación y transmisión de caracteres en plantas. Es a partir de 1900 que un grupo importante de genetistas, encabezados por Thomas Morgan (1810-1915), infieren la existencia de genes y crean mapas de localización en los cromosomas de Drosophila melanogaster.
Aunque el comienzo de siglo XX encuentra a Chile con una fauna y flora relativamente bien conocidas y con la aceptación intelectual de las ideas de Darwin, era necesario mejorar la capacidad científica tanto en universidades como en centros educacionales, lo cual incidió en un progreso en el estudio y enseñanza de la genética. La Escuela de Medicina de la Universidad de Chile fue el locus de la mayor parte de la incipiente investigación efectuada en el momento. El interés del gobierno en mejorar la calidad de la medicina efectuada en el país, se tradujo en la contratación del médico italiano Giovanni Noé (1876-1946) en 1912. Noé era profesor de Zoología Médica en la Universidad de Roma, y discípulo del zoólogo italiano Giovanni Grassi, involucrado en la descripción del ciclo de vida de Plasmodium. Noé tuvo un profundo impacto sobre sus estudiantes, siendo responsable de la enseñanza de la zoología, morfología y genética al nivel de las universidades de Europa. Aunque su contribución a la biología chilena no ha sido adecuadamente reconocida, Noé preparó el camino para la primera generación de genetistas en Chile (Medel 2008). Mientras tanto, $\mathrm{y}$ en paralelo con las enseñanzas de Noé en Chile, se lograban importantes avances cuantitativos en genética en los países desarrollados, fundamentalmente a través de los trabajos teóricos de Ronald Fisher (1890-1962), John B.S. Haldane (1892-1964) y Sewall Wright (1889-1988). Desde entonces, el énfasis en el estudio de la evolución se desplazó hacia programas de investigación en poblaciones naturales en busca de los mecanismos que daban cuenta del cambio evolutivo descubierto por Darwin. El quehacer ya no estaba centrado en la discusión de la existencia o no de la evolución sino en los mecanismos genéticos que posibilitaban o restringían el cambio evolutivo.

Después de la Segunda Guerra Mundial, el desarrollo de la biología y medicina en Chile siguió casi invariablemente el modelo anglosajón de desarrollo. Apoyada en la influencia de la teoría cromosómica de la herencia postulada por Morgan a comienzos de siglo y gracias a las contribuciones de los norteamericanos Clarence McClung y Leylard Stebbins, probablemente la citogenética fue la primera disciplina que completó su desarrollo en Latinoamérica (Cruz-Coke 1999). La contribución más conspicua de esta disciplina al área naturalista consistió en la identificación de los cromosomas de diversas especies de plantas, 
mamíferos, reptiles y anfibios con propósitos sistemáticos y de clasificación, ambos aspectos que implícitamente daban cuenta de fenómenos de formación de especies en los sistemas naturales. Los excitantes avances en biología evolutiva, articulados en la Síntesis Moderna fueron propagados a Chile gracias a la visita de Theodosius Dobzhansky en 1956. La estadía de Dobzhansky tuvo una profunda influencia sobre un joven estudiante que posteriormente sería el científico más influyente en la historia de la biología evolutiva en Chile. Danko Brncic (1922-1998) efectuó una estadía en el laboratorio de Dobzhansky en la Universidad de Columbia, donde tuvo contacto de primera fuente con las diversas preguntas y aproximaciones en el estudio de la genética ecológica en Drosophila. La diversificación de Drosophila, producto de la peculiar biogeografía de Chile, proporcionó un adecuado sustrato biológico para el desarrollo de un programa de investigación local sobre la evolución del grupo en el país. Es así como Brncic se involucró activamente en el desarrollo de la Teoría Sintética de la Evolución (e.g., Brncic 1960), llegando a liderar la investigación en genética de poblaciones no sólo en Chile sino en Sudamérica. Luego de su retorno a Chile, ocurrió una explosión de investigaciones en genética básica y aplicada, citogenética, historia natural y sistemática, evolución experimental y biología de poblaciones. La influencia de Brncic conjuntamente con la emergente escuela de citogenetistas en Chile dieron el impulso definitivo a los estudios de biología evolutiva en el país, fundándose la Sociedad de Genética de Chile en 1964. Una de las misiones de esta sociedad científica fue promover el avance y enseñanza de la genética en niveles de pregrado y postgrado. Las huellas de esta iniciativa son identificables hasta el día de hoy, cuando varias sociedades científicas naturalistas han proliferado, y el estudio de la biología evolutiva forma parte importante de los programas de investigación en el país.

\section{AGRADECIMIENTOS}

Agradecemos a Guillermo D'Elía por su invitación a participar con una contribución al número especial de Gayana dedicado a la obra de Charles Darwin. Carezza Botto efectuó importantes comentarios a una versión preliminar de este manuscrito. Este trabajo fue desarrollado con subsidios indirectos del Proyecto Anillo PBCT 34/2006 a RM y FONDECYT 1061256 a AV.

\section{BIBLIOGRAFÍA}

Bacigalupo, J. \& D. Yudilevich. 1998. Andrés Bello y la visita de Charles Darwin a Chile. Ciencia al Día 1:1-11.

BRncic, D. 1960. Cien años de Darwinismo. Anales de la Universidad de Chile 117-118: 138-145.

Cruz-CoKE, R. 1999. Historia de la genética latinoamericana en el siglo XX. Revista Médica de Chile 127:1524-1532.

DARWIN, C. 1845. The voyage of the Beagle. Journal of researches into the natural history and geology of the countries visited during the voyage of H.M.S. Beagle around the world, under the command of Capt. Fitz Roy, R.A. 2nd Edition. Murray, London. $536 \mathrm{pp}$.

DARWIN, C. 1859. On the origin of species by means of natural selection, or the preservation of favoured races in the struggle for life. $6^{\text {th }}$ Edition 1872. Carlton House, New York. 372 pp.

Del Barrio, P. 1855. Sobre los temblores de tierra i sus efectos en general i en especial los de Chile. Anales de la Universidad de Chile 1855:583625.

Dомеүко, I. 1874. Cuarto apéndice al tratado de mineralojia i reino mineral de repúblicas vecinas. Anales de la Universidad de Chile 1874:99-152.

ELDREDGE, N. 2005. Darwin: discovering the tree of life. Norton, New York. 288 pp.

ELDREDGE, N. 2009. Experimenting with transmutation: Darwin, the Beagle, and evolution. Evolution: Education and Outreach 2:35-54.

FuenzalidA, A. 1934. A cien años de un viaje famoso: Darwin en Chile. Prensa de la Universidad de Chile, Santiago. 49 pp.

Hodge, C. 1874. What is Darwinism? Scribner \& Armstrong, New York. 178 pp.

KuHN, T. 1962. The structure of scientific revolutions. University of Chicago Press, Chicago. 172 pp.

Malthus, T.R. 1826. An essay on the principle of population. $6^{\text {th }}$ Edition. Murray, London.

Márquez Breton, B. 1982. Orígenes del Darwinismo en Chile. Editorial Andrés Bello, Santiago de Chile. $111 \mathrm{pp}$.

Medel, R. 2008. The evolution of evolutionary thinking in Chile. Evolution: Education and Outreach 1:318-322.

PAPP, D. 1977. Ideas revolucionarias en la ciencia: su historia desde el Renacimiento hasta promediar el siglo XX. Tomo II. La edad de las grandes síntesis. El siglo XIX. Editorial Universitaria, Santiago de Chile. 321 pp.

SalazAR G. \& J. Pinto. 1999. Historia contemporánea de Chile I. Estado, legitimidad, ciudadanía. LOM, Santiago de Chile. 311 pp.

Tamayo H. M. 2004. Las teorías biológicas evolutivas 
en textos de estudio en Chile. Tesis doctoral, Universidad de Granada. Granada, España

TAmayo H. M. \& F. GonzÁlez. 2005. La enseñanza de la Evolución en Chile: Una historia de conflictos documentada en textos de estudio. VII Congreso Enseñanza de las Ciencias. pp. 1-5.

UrzÚA, C. 2009. Chile en los ojos de Darwin: veinte meses de viaje por el país físico y humano. Ediciones B, Santiago de Chile. 197 pp.
Villalobos, S., O. Silva, F. Silva \& P. Estelle. 1974. Historia de Chile. Tomo 3. Editorial Universitaria, Santiago de Chile. 561 pp.

Vitale, L. 1991. Interpretación marxista de la historia de Chile. Volumen III. Editorial LOM, Santiago de Chile. 173 pp.

Yudilevich, D. \& E. Castro (Eds.). 1996. Darwin en Chile: viaje de un naturalista alrededor del mundo. Editorial Universitaria, Santiago de Chile. 